\title{
Physiopathology of the diabetic bladder
}

\author{
Tatiana Bolgeo ${ }^{1}$, Antonio Maconi ${ }^{1}$, Marinella Bertolotti ${ }^{1}$, Annalisa Roveta ${ }^{1}$, Marta Betti ${ }^{1}$, \\ Denise Gatti ${ }^{1}$, Carmelo Boccafoschi ${ }^{2}$ \\ ${ }^{1}$ Azienda Ospedaliera SS. Antonio e Biagio e C. Arrigo, Alessandria, Italy; \\ ${ }^{2}$ Clinica Città di Alessandria Policlinico di Monza, Alessandria, Italy.
}

\begin{abstract}
Summary Objective: To investigate the incidence of diabetic cystopathy in relation to age, gen-
\end{abstract} der, type of diabetes, duration of diabetic disease and clinical evidence of peripheral neuropathy and to analyze the physiopathology of the various forms of diabetic cystopathy due to sensory impairment, motor-sensory impairment, motor impairment and hyperreflexia.

Materials and methods: In a retrospective multicenter cohort study the medical records of a cohort of 126 diabetic patients with (128 patients) or without (48 patients) urological symptoms were analyzed. Patients were observed at the Città di Alessandria Clinic of Policlinico di Monza and/or at the outpatient clinic of Alessandria Hospital from June 2018 to June 2020. The study excluded patients with central and/or peripheral neuropathy, spina bifida (mylomeningocele or meningocele) or spina bifida occulta; with persistent urinary infections; in anticholinergic treatment for enteric dysfunctions; in medical treatment for cervical-prostatic-urethral obstruction; with vaginal and/or rectal prolapse of II, III, IV degree; with previous spinal or pelvic surgery including radical prostatectomy, Wertheim hysterectomy or colorectal surgery. All the patients were studied with computed tomography (CT) scan of the urinary tract, voiding cystourethrography (VCUG), uroflowmetry, cystomanometry with intrinsic pressure assessment and compliance evaluation, electromyography (EMG) of the anal sphincter, pressure flow analysis, urethral pressure profile and, when advised, pharmacological tests.

Results: Out of 126 diabetic patients, 48 did not show any signs or symptoms of urine voiding dysfunction; 30 were men and 18 women with an average age of 62.6 years; 20 had type I diabetes and were in treatment with insulin and 28 type II diabetes treated with oral hypoglycemic medication. The remaining 78 patients ( 48 men and 30 women), with an average age of 64.8 years, presented urological symptoms; 31 had type I diabetes and 47 had II type diabetes. Conclusions: Diagnosis of the various forms of diabetic cystopathy and early treatment decreases complications and consequently accesses to outpatient facilities and hospital admissions, resulting in an improved quality of life.

KEY WORDS: Diabetic neuropathy; Neurological bladder; Diabetes mellitus; Urodynamics.

Submitted 13 October; Accepted 16 November

\section{INTRODUCTION}

Changes in diabetic patients' bladder functions are part of the wider field of autonomic diabetic neuropathy, but from the neuropathophysiological point of view, the importance of the condition is not yet entirely specified. Diabetic neuropathy is the most common and disturbing complication of diabetes, it leads to the highest morbidity and mortality rates, therefore producing an enormous economic burden in treatments. Clinical evaluation and treatment of diabetic peripheral neuropathy has multiple options and patients with diabetic neuropathy should be screened for autonomic neuropathy, as there is a high degree of coexistence of the two complications (1). Nevertheless, this disease is also of great interest in relation to the high morbidity and occasional fatal consequences (2). This assumption motivated the investigation to verify the rate of diabetic cystopathy in relation to the type of diabetes and the correlation or not with a neuro-urological subjective symptomatology (3). While diabetes as a metabolic disease is of easy recognition, diabetic cystopathy develops insidiously and symptoms may not appear until the disease is already in an advanced stage (1). The consequences of diabetes on bladder function have been known since 1864 (4), but subsequently in literature often conflicting data were reported, due to both the selection of patients according to different diabetic and urological criteria, as to the occasional diversity in definitions of diabetic cystopathy, not always compliant and identical. In fact, the incidence of "bladder dysfunction" in diabetic patients varies from $37 \%$ to $67 \%$ (5). Before presenting the study and making assessments on the incidence of diabetic cystopathy it is relevant to consider some predictive factors such as age, gender, type and degree of diabetes, factors that may have caused a (unintended) patient selection, linked to the characteristics of the study population. Only with this foresight, it will be possible to attempt comparative assessments between the various authors who have so far treated the problem. Some studies include in the definition of diabetic cystopathy sensory impairment (1), others sensory and sensory-motor impairment (6), others in addition to the previous also consider the apparently isolated motor impairment of the detrusor (hyperreflexia, contractility devoid) without compulsory and prevailing sensory impairment (7).

In the present case study, patients suffering from diabetic cystopathy (in the broad interpretation of the term as diabetic autonomic neuro-bladder) were considered as patients that had sensitive impairment, sensitive-motor impairment, isolated motor impairment and detrusor "hyperreflexia". Particular attention was paid to identify 
other coexistent illnesses and diseases, especially the presence of cervical-prostatic-urethral obstructions. The diagnostic tools used to evaluate the type of diabetic cystopathy include urodynamic evaluation (uroflowmetry, cystomanometry with intrinsic pressure assessment and compliance evaluation, electromyography (EMG) of the anal sphincter, pressure-flow study, urethral pressure profile and when advised pharmacological tests), ultrasound and computed tomography (CT) scan of the urinary tract and voiding cysto-urethrography.

When assessing the association between autonomic neuropathy and cervical-prostatic obstruction, differential urodynamic diagnosis can be difficult and maybe sometimes even impossible. Conversely after the removal of the obstruction (clinically and urodynamically proven) it is easier to evaluate bladder sensitivity and residual detrusorial contraction function associated to diabetic cystopathy. During follow up, ultrasound examinations were used to assess the post-void residue. Patients were separately evaluated according to the type of diabetes and time-period of onset or first clinical diagnosis. From literature, it does not emerge a precise nosology of diabetic neuropathies and even less of diabetic cystopathy that was generically considered as an alteration of bladder function that can be independent or linked to autonomic visceral neuropathy.

The aim of this study was to assess in a cohort of patients the incidence of the above described bladder dysfunctions (diabetic cystopathy) in relation to predictive factors such as age, gender, type and timing of diabetic onset.

\section{Methods}

In this retrospective multicentre cohort study, the clinical records of 126 diabetic patients treated at "Città di Alessandria Clinic" and/or at out-patient clinic of the Alessandria Hospital from June 2018 to June 2020 were reviewed.

Exclusion criteria included: patients with central and/or peripheral neuropathy (Parkinson. multiple sclerosis, stroke, etc.); patients with Spina Bifida (mylomeningocele or meningocele) or Spina Bifida Occulta; patients with persistent urinary infections; patients in anticholinergic treatment for enteric dysfunctions; patients in medical treatment for cervical-prostatic-urethral obstruction; patients with vaginal and/or rectal prolapse of II, III, IV degree; patients with previous spinal or pelvic surgery including radical prostatectomy, Wertheim hysterectomy or colorectal surgery.

\section{Results}

Out of the 126 diabetic patients observed, 48 did not show signs or symptoms of dysfunctional voiding; 30 were men and 18 women; the average age was 62.2 years; 20 presented type I diabetes and were on treatment with insulin and 28 had type II diabetes and were on oral hypoglycemic treatment. The remaining 78 patients showed urological symptoms; 48 were men and 30 women; average age was 64.8 years; 31 presented type I diabetes and 47 type II diabetes.

The 78 diabetic patients with urological symptoms were
Table 1.

Results of urodynamic evaluation in patients with diabetes type I and II.

\begin{tabular}{|lccc|}
\hline Urodynamic results & Type I diabetes & Type II diabetes & \\
\hline Sensory impairment & 11 & 12 & \\
\hline Motor - Sensory impairment & 5 & 5 & \\
\hline Motor impairment & 5 & 6 & \\
\hline Hyperreflexia without obstruction & 1 & 8 & Tot 53 \\
\hline & $\mathbf{2 2}$ & $\mathbf{3 1}$ & \\
\hline Obstruction & 4 & 13 & \\
\hline Normal & 5 & 3 & Tot 78 \\
\hline Totale & $\mathbf{3 1}$ & $\mathbf{4 7}$ & \\
\hline
\end{tabular}

divided into subgroups according to the type of diabetes and the presence or not of diabetic cystopathy that was differentiated in its various forms by urodynamic evaluation (Table 1).

Sensory impairment was identified in 11 patients with type I diabetes and in 12 patients with type II diabetes (a prostatic obstruction not pharmacologically treated was associated in 2 patients with type I diabetes and in 3 patients with type II diabetes, respectively). A mixed motor-sensory impairment was identified in 5 patients with type I diabetes and in 5 patients with type II diabetes (in 2 of the latter, a non-pharmacologically treated cervical-prostatic obstruction was also identified). Isolated motor impairment without sensory implication were identified in 5 type I diabetic patients and in 6 type II diabetic patients (in 1 of the latter a non-pharmacologically treated cervical-prostatic obstruction was also present).

In total, the association between diabetic cystopathy and cervical-prostatic-urethral obstructive disease was present, in 8 patients. The diagnosis of bladder outlet obstruction was conveyed on clinical and radiological data, pressure flow studies and urethral pressure profilometry (UPP), although in presence of hypocontractility or acontractility of the detrusor the flow-pressure relationship was not very significant. In 5 out of 8 cases, postvoiding residue was more than 10\% (sign of decompensation). In these cases the presence of neuropathy was outstanding because when they were urodynamically studied after the removal of the cervical-prostatic obstruction hypo-contractility or a-contractility of the detrusor responded to treatment with parasympatheticmimetic drugs.

Detrusor hyperreflexia (with unstable contractions but with normal urethral resistances) was present in 1 patient with type I diabetes and in 8 type II diabetic patients. Urodynamic examination was essential to identify this group, because it allowed to exclude obstructive anatomical and/or functional factors.

The urodynamic examination also played an important role in evaluating a group of 17 patients with cervicalprostatic-urethral obstruction without any sign of diabetic cystopathy, these patients evidenced normal bladder sensitivity but detrusor hyper-contractility.

In 8 other patients ( 5 with type I and 3 with type II diabetes) the urodynamic evaluation did not identify any impairment allowing to consider these patients as normal for bladder function. 
Altogether, diabetic cystopathy was present in $71 \%$ $(22 / 31)$ of patients with type I diabetes and 66\% (31/47) of patients with type II diabetes.

Peripheral somatic neuropathy was clinically present in 20 out 78 patients with urological symptoms. Fifteen of these $(75 \%)$ also had diabetic cystopathy, while only $65 \%$ of the remaining 58 patients without clinically detectable signs and symptoms of somatic neuropathy, evidenced bladder dysfunctions indicative of diabetic cystopathy. Only in 15 of patients with diabetic cystopathy (about 28\%) a peripheral somatic neuropathy was clinically demonstrated.

To evaluate the relationship between the duration of diabetic disease and the incidence of autonomic cystopathy, the patients included in the present study, were divided into two groups according to whether the diabetic disease had been diagnosed for less than 5 years ( 28 patients) or more than 5 years (50 patients).

The average age of the two groups was not significantly different being respectively 62 and 66 years.

Of the recently diagnosed patients, 16 out of 28 (57\%) presented diabetic cystopathy, while 37 out of 50 (74\%) of the remaining with long-lasting diabetes showed signs of cystopathy.

In $37 / 53(70 \%)$ of the patients with diabetic cystopathy, the diabetic disease was present from more than 5 years.

\section{Discussion}

The multiple aspects of the diabetic bladder can be due to both the variety of neurological impairments specific of the disease that affect the nervous system at various levels, as to the altered pathophysiology of the organ affected by neuro-impairment (8). The autonomic and somatic diabetic neuropathy has probably a multifactorial origin. The multiplicity of locations and extent of impairment can therefore explain why there are different types of diabetic neurological bladder (9).

In our case study of diabetic patients with urological symptoms, a neurogenic bladder dysfunction was identified in $66 \%$ of cases.

The total of the patients included 23 patients who presented exclusively sensory impairment, 10 patients with motor-sensory impairment, 11 patients with exclusive motor impairment and 9 patients with hyperreflexia (without obstruction) (Figures 1-4).

Seventeen of the remaining patients presenting with urological symptoms (22\%) had cervical-prostatic obstruction and 8 patients (10\%) were urodynamically normal.

Diabetic cystopathy, as neurogenic bladder dysfunction caused by autonomic diabetic neuropathy, occurs with remarkable frequency ( $42 \%$ of the 126 diabetic patients studied) particularly if urological symptoms are present (68\% of this group of 78 patients).

In our series, type of diabetes (insulin dependent versus non-insulin dependent) was not associated with an higher risk of diabetic cystopathy, that was observed in $71 \%$ of symptomatic patients with type I diabetes and $66 \%$ of symptomatic patients with type II diabetes.

Consequently, it seems that the insulin dependence of diabetes is not the determining factor of onset of autonomic cystopathy. On the contrary, Frimodt-Møller (10),
Figure 1.

Urodynamic trace of a case of diabetic cystopathy with impairment restricted only to the sensory pathways (modified by Boccafoschi C, Maurel A Appunti sui parametri e le metodiche in urodinamica. Minerva Medica; 1986)

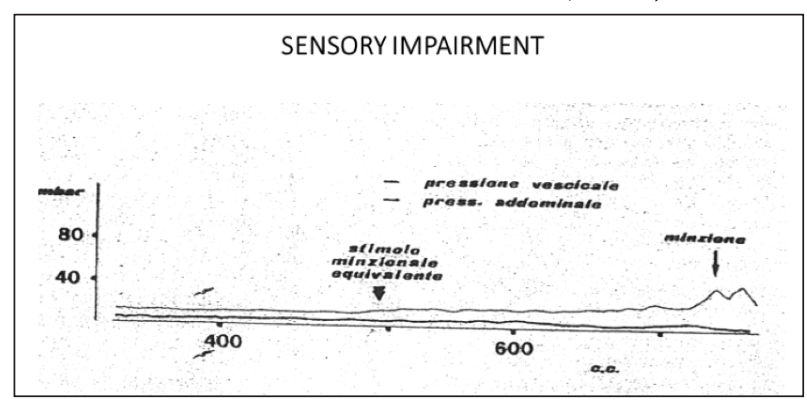

Figure 2.

Urodynamic trace in a case of diabetic cystopathy with impairment of the motor-sensory pathways (modified by Boccafoschi C, Maurel A Appunti sui parametri e le metodiche in urodinamica. Minerva Medica; 1986)

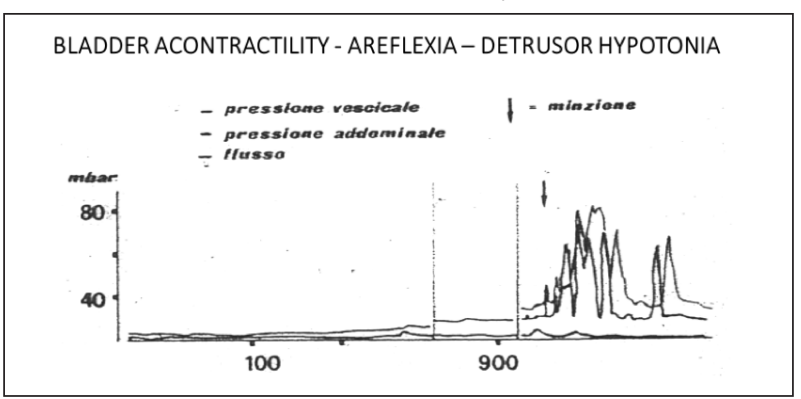

\section{Figure 3.}

Urodynamic trace in a case of diabetic cystopathy with impairment restricted only to the motor pathways (modified by Boccafoschi C, Maurel A Appunti sui parametri e le metodiche in urodinamica. Minerva Medica; 1986).

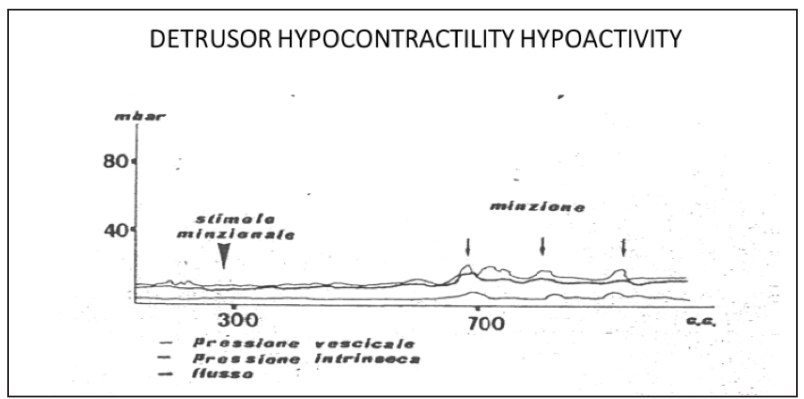

Figure 4.

Urodynamic trace in a case of diabetic cystopathy with detrusor hyperreflexia (modified by Boccafoschi C, Maurel A Appunti sui parametri e le metodiche in urodinamica. Minerva Medica; 1986).

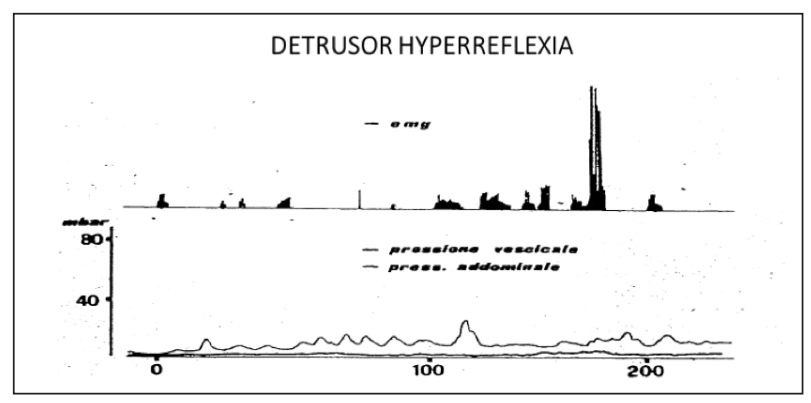


evaluating a different series, obtained different results showing a higher incidence of cystopathy in insulin dependent diabetics (48\%) than in diabetics on oral hypoglycemic treatment or treated only with diet $(25 \%)$. Conversely, their series was not comparable with our series because of lower mean age of patients, higher prevalence of patients treated with insulin (80\%) and longer duration of the diabetic disease.

The most relevant neurological disease related to diabetes in addition to autonomic neuropathy is peripheral somatic neuropathy, with the association between diabetic cystopathy and peripheral neuropathy reported in percentages varying from $14 \%$ to $80 \%$ (11-13).

The association between peripheral neuropathy and diabetic cystopathy was demonstrated in 19\% of the cases with symptomatic diabetes. The rate of cystopathy was high in neuropathic patients (75\%) but this was not true for the opposite. In fact, only $28 \%$ of patients with diabetic cystopathy showed clinically demonstrable peripheral neuropathy as if the impairment of the visceral autonomic nervous system precedes in time that of the peripheral autonomic system or that of the somatic nerves.

This is confirmed by the data relating the rate of cystopathy (and neuropathy) and the duration of the diabetic disease, especially if it is above 5 years. Cystopathy is in fact, present in $74 \%$ of diabetic patients that have been afflicted with the disease for more than 5 years, compared to only $57 \%$ of the patients who have been afflicted with diabetes for less than 5 years.

\section{Conclusions}

In literature, up to the present day, all the studies have addressed symptomatic and non-symptomatic diabetic patients from an urological perspective.

Urodynamic techniques can successfully study the pathophysiology of the diabetic bladder and allow identifying four types of diabetic cystopathy: sensory pathway impairment, motor-sensory pathway impairment, motor pathway impairment and impairment to control detrusorial reflex resulting in hyperreflexia. The urodynamic evaluation can also identify cases of simple cervical-prostatic obstruction without signs of diabetic cystopathy. Nevertheless, particular problems arise when there is an association between diabetic cystopathy and cervical-prostatic-urethral obstruction. The urodynamic evaluation of detrusor contractility function, urethral function and bladder sensitivity makes it possible to convey a precise diagnosis, even if in a number of cases it becomes necessary to repeat the test after having surgically removed the cervical-prostatic-urethral obstruction. In the assessment of bladder sensitivity, the urodynamic evaluation is limited and it may be useful to integrate it with the measurement of the threshold of electrical perception or with the study of evoked potentials. The complexity of nerve structures that control bladder functioning and the possibility that diabetes can impair them with multiple mechanisms (dis-metabolic, toxic, micro- or macro- angiopathic) and at multiple levels of the central and peripheral nervous system, makes questionable the claim that the impairment responsible for diabetic cystopathy is due exclusively to peripheral impairment. Conversely, being central impairment alone not confirmed, mixed forms should be accepted as the more frequent.

In the future, it could be worthwhile the use of dynamic brain Magnetic Resonance studies.

These results may have clinical applicability in identifying patients with diabetic cystopathy and guide patient counselling with treatment decision-making.

\section{REFERENCES}

1. Feldman EL, Callaghan BC, Pop-Busui R, et al. Diabetic neuropathy. Nat Rev Dis Primers. 2019; 5:42.

2. Deli G, Bosnyak E, Pusch G, et al. Diabetic neuropathies: diagnosis and management. Neuroendocrinology. 2013; 98:267-80.

3. Ziegler D. Painful diabetic neuropathy: treatment and future aspects. Diabetes Metab Res Rev. 2008; 24 Suppl 1:S52-7.

4. Tanik N, Tanik S, Albayrak S, et al. Association between overactive bladder and polyneuropathy in diabetic patients. Int Neurourol J. 2016; 20:232-239.

5. Duby JJ, Campbell RK, Setter SM, et al. Diabetic neuropathy: an intensive review. Am J Health Syst Pharm. 2004; 61:160-73.

6. Bossi L, Caffaratti E. Bladder complications in neuropathic diabetic patients. Clinical and radiological study. Minerva Radiol. 1968; 13:40-7.

7. Bradley WE. Cerebro-cortical innervation of the urinary bladder. Tohoku J Exp Med. 1980; 131:7-13.

8. Bolgeo T. Caring for bladder dysfunctions in patients with Parkinson's disease. Arch Ital Urol Androl. 2011; 83:112-5.

9. Boccafoschi C, Maurel A. Appunti sui parametri e le metodiche in urodinamica. Minerva Medica; 1986.

10. Frimodt-Møller C. Diabetic cystopathy: epidemiology and related disorders. Ann Intern Med. 1980; 92:318-21.

11. Freeman R. Diabetic autonomic neuropathy. Handb Clin Neurol. 2014; 126:63-79.

12. Carone R, Frea B, Tizzani A, Borgno M. Neurologic bladder in diabetes. Etiopathogenetics and treatment. Minerva Urol. 1979; 31:91-3.

13. Kiani J, Moghimbeigi A, Azizkhani H, Kosarifard S. The prevalence and associated risk factors of peripheral diabetic neuropathy in Hamedan, Iran. Arch Iran Med. 2013; 16:17-9.

\author{
Correspondence \\ Tatiana Bolgeo \\ Antonio Maconi \\ Marinella Bertolotti \\ Annalisa Roveta \\ Marta Betti \\ Denise Gatti \\ Azienda Ospedaliera SS Antonio e Biagio e C. Arrigo, Alessandria (Italy) \\ Carmelo Boccafoschi, MD (Corresponding Author) \\ cboccafoschi@virgilio.it \\ Clinica Città di Alessandria Policlinico di Monza, Alessandria (Italy)
}

